\title{
The Effectiveness and Value of Patisiran and Inotersen for Hereditary Transthyretin Amyloidosis
}

\section{A Summary from the Institute for Clinical and Economic Review's Midwest Comparative Effectiveness Public Advisory Council}

\author{
Kristin Mickle, MPH; Karen E. Lasser, MD, MPH; Jeffrey S. Hoch, PhD; Lauren E. Cipriano, PhD; \\ William B. Dreitlein, PharmD, BCPS; and Steven D. Pearson, MD, MSc
}

$\mathrm{H}$ ereditary transthyretin amyloidosis (hATTR) is a rare, autosomal dominant disease caused by the misfolding of the liver protein transthyretin (TTR). Patients ultimately develop TTR depositions across multiple body systems and organs leading most prominently to progressive polyneuropathy or cardiac dysfunction, with many patients experiencing both. ${ }^{1}$ Polyneuropathy associated with hATTR significantly impairs motor, autonomic, and sensory nerve function. Initial neuropathic pain is followed by progressive motor weakness, ultimately leading to the inability to walk. Gastrointestinal impairment due to autonomic neuropathy is also disabling, and it can progress to later-stage wasting and cachexia. ${ }^{2}$ TTR deposition in cardiac tissue can result in thickening of ventricular walls, diastolic dysfunction, arrhythmia, and heart failure. ${ }^{3}$

Approximately 10,000 people worldwide have been diagnosed with hATTR-related neuropathy, of which 3,000-3,500 patients are in the United States. The incidence of cardiomyopathy due to hATTR is largely unknown because of varying estimates of the proportion of patients who develop clinical disease (7\%-80\%), although prevalence is known to increase with advancing age. The median age at onset of symptoms is 68.1 years, and the median survival time from diagnosis is 5-15 years; survival is shorter for individuals with cardiac involvement (2.5-4 years). ${ }^{2}$

Standard treatment for hATTR includes diflunisal and liver transplantation, but neither approach reverses the damage caused by TTR deposits, and both treatments are restricted to relatively healthy patients due to safety and mortality risks. The Institute for Clinical and Economic Review (ICER) recently conducted a review of 2 new first-in-class drugs for hATTR: patisiran (Onpattro, Alnylam Pharmaceuticals) and inotersen (Tegsedi, Akcea Therapeutics). Both drugs aim to reduce TTR by targeting the RNA that produces the misfolded protein, either by enhancing RNA degradation (inotersen) or by reducing its production (patisiran). The U.S. Food and Drug Administration (FDA) approved patisiran on August 10, 2018; when ICER concluded its assessment in September 2018, inotersen was still undergoing FDA review. Here, we present a summary of the systematic literature review, cost-effectiveness

J Manag Care Spec Pharm. 2019;25(1):10-14

Copyright $\odot 2019$, Academy of Managed Care Pharmacy. All rights reserved. analyses, and policy discussion with key stakeholders regarding the overall value of these RNA-targeted therapies. The full report is published on ICER's website at www.icer-review.org. ${ }^{5}$

\section{Summary of Findings}

\section{Clinical Effectiveness}

Our review compared the clinical effectiveness, safety, and economic effects of patisiran and inotersen monotherapy for patients with hATTR. Because of differences in populations (e.g., race, geographic region, and disease severity) and the use of 2 different novel neuropathy outcome measures, ${ }^{3,6}$ we did not directly compare the 2 drugs through meta-analysis; comparisons were possible only to the supportive care arm in the respective trials. Complete details on ICER's systematic literature search and protocol, including search strategy and PICOTS criteria, are available on ICER's website.

Our review included 3 references that reported inotersen safety and efficacy data from the 15-month randomized, double-blind, phase 3 NEURO-TTR trial and 1 publication that reported cardiac data from a single-arm, open label study. ${ }^{6-9}$ Patients in NEURO-TTR $(n=172)$ were in earlier stages of disease and had neuropathy-predominant symptoms of hATTR; those with previous liver transplants or signs of heart failure were excluded. After 15 months of treatment, inotersen slowed the progression of polyneuropathy (assessed by mNIS +7 score) relative to placebo and stabilized neuropathy-related quality of life as measured by the Norfolk-QOL-DN score. For these co-primary outcomes, $36.5 \%$ of inotersen patients (risk/proportion difference $=17 \%, 95 \%$ confidence interval $[C I]=2.4-32.1$ vs. placebo) showed improved mNIS + 7 scores, and half reported improved quality of life (risk/proportion difference $=23.1 \%, 95 \% \mathrm{CI}=7.0-39.2$ vs. placebo). Open-label extension data suggest sustained delay of polyneuropathy progression. ${ }^{6}$ There was no evidence of improvement versus placebo in echocardiographic measures with inotersen treatment after 15 months. ${ }^{6}$

Five (4\%) deaths were reported among patients in the NEURO-TTR trial, all of which occurred in the inotersen group; 1 death was considered possibly related to inotersen. In addition, platelet counts below 140,000 cells $/ \mathrm{mm}^{3}$ were reported in $54 \%$ of inotersen patients versus $13 \%$ of placebo patients, and three (3\%) cases of glomerulonephritis occurred among 
inotersen recipients. ${ }^{6}$ The final product label of inotersen included an FDA black box warning for thrombocytopenia and glomerulonephritis. ${ }^{10}$

For patisiran, 15 references met our inclusion criteria, $3,11-24$ including 2 peer-reviewed publications reporting results from the 18-month randomized, double-blind, phase 3 APOLLO trial. ${ }^{3,25}$ Patients in the APOLLO trial tended to be in intermediate stages of disease; exclusion criteria were similar to the NEURO-TTR trial. APOLLO results demonstrated a statistically significant mean improvement in neurologic function (primary outcome) and neuropathy-related quality of life (secondary outcome) with patisiran treatment compared with placebo. Seventy percent of patisiran patients reported stable or improved neuropathy stage (odds ratio $[\mathrm{OR}]=39.9,95 \% \mathrm{CI}=11.0-144.4$ vs. placebo), and $51 \%$ of patisiran patients showed neurological improvement by mNIS +7 score $(\mathrm{OR}=10.0,95 \% \mathrm{CI}=4.4-22.5$ vs. placebo). Post hoc analyses suggest a decreased risk of the composite endpoint of all-cause mortality and hospitalization among patients with cardiac involvement. ${ }^{25}$

Deaths were balanced between the patisiran (5\%) and placebo (8\%) arms of the study. ${ }^{3}$ Treatment discontinuations due to adverse events were more common among placebo than patisiran patients. The most common adverse events reported in APOLLO were peripheral edema and infusion-related reactions; however, 4 serious adverse reactions of atrioventricular heart block occurred in patients who received patisiran.

\section{Limitations of the Clinical Evidence}

This evidence base is limited by the common limitations of rare disease evidence, including a small patient population, limited disease-specific clinical measures, clinical trial design challenges, and a lack of long-term safety and efficacy data. The patients enrolled in these studies had neuropathic clinical manifestations and are thus not representative of the broader patient population in which cardiac manifestations predominate. In addition, functional outcome measures were quite limited in both studies. Ultimately, for both medications, we were unable to quantify the magnitude of clinical benefit in polyneuropathy measured by the mNIS + 7 score and neuropathy-related quality of life without validated thresholds for meaningful clinical change.

\section{Long-Term Cost-Effectiveness}

We also estimated the cost-effectiveness, using quality-adjusted life-year (QALY) as the outcome of inotersen and patisiran monotherapy versus supportive care, under a Markov model specific to each trial population. The model was developed with 2 base cases-a health care sector and a societal perspective-under ICER's ultra-rare disease value framework, with costs and outcomes discounted at 3\% annually. ${ }^{26}$ The economic model for each drug included 6 disease states: 1 each for familial amyloid neuropathy (FAP) stage 1, 2, and 3 and

\begin{tabular}{|c|c|c|c|}
\hline TABLE 1 & \multicolumn{3}{|c|}{$\begin{array}{l}\text { Health Care Sector Perspective } \\
\text { Results for Inotersen and Patisiran } \\
\text { Cost-Effectiveness }\end{array}$} \\
\hline & \multicolumn{2}{|c|}{ Discounted } & \multirow{2}{*}{\begin{tabular}{|c|} 
Versus \\
Supportive Care \\
Incremental \\
Cost-Effectiveness \\
Ratio (QALYS) \\
\end{tabular}} \\
\hline & Total Costs, \$ & QALYs & \\
\hline \multicolumn{4}{|c|}{ Health care sector perspective } \\
\hline Inotersen & $1,507,450$ & 4.54 & \multirow{2}{*}{$1,730,000$} \\
\hline Supportive care & 329,858 & 3.86 & \\
\hline Patisiran & $3,173,084$ & 6.54 & \multirow{2}{*}{835,000} \\
\hline Supportive care & 312,062 & 3.11 & \\
\hline \multicolumn{4}{|c|}{ Modified societal perspective } \\
\hline Inotersen & $1,608,862$ & 4.54 & \multirow{2}{*}{$1,730,000$} \\
\hline Supportive care & 431,261 & 3.86 & \\
\hline Patisiran & $3,355,304$ & 6.54 & \multirow{2}{*}{853,000} \\
\hline Supportive care & 432,031 & 3.11 & \\
\hline \multicolumn{4}{|c|}{ QALY $=$ quality-adjusted life-year. } \\
\hline
\end{tabular}

corresponding states for each FAP stage with severe cardiac involvement. Potential cardiac treatment benefits were assessed only in scenario analysis, given a lack of data on these effects from the trials. Full details on ICER's cost-effectiveness analysis and model are available on ICER's website. ${ }^{5}$

The price of both drugs in the U.S. market, net of anticipated discounts and rebates, is $\$ 345,000 .{ }^{27}$ The economic model found that the incremental cost-effectiveness ratios for inotersen were $\$ 1,730,000$ per QALY gained in the health care and societal perspectives (Table 1). The incremental cost-effectiveness ratios for patisiran were $\$ 835,000$ per QALY gained from a health care perspective and $\$ 853,000$ per QALY gained from a societal perspective.

\section{Limitations of the Cost-Effectiveness Model}

This model relies on several assumptions and extrapolation from our current knowledge. Costs and quality of life measures have not been studied comprehensively for the specific trial populations; therefore, we assumed similarities between the NEURO-TTR and APOLLO populations and people in other studies of hATTR. Also, we were limited in measures of effectiveness for hATTR to those measures that were captured in the clinical trials as outcomes (i.e., FAP stage changes), as well as in the types of measures that could be linked to quality of life.

\section{Policy Discussion}

The Midwest Comparative Effectiveness Public Advisory Council (Midwest CEPAC) is one of the independent appraisal committees convened by our institute to engage in the public deliberation of the evidence on clinical and cost-effectiveness of health care interventions. The Midwest CEPAC is composed of medical evidence experts, including practicing clinicians, 


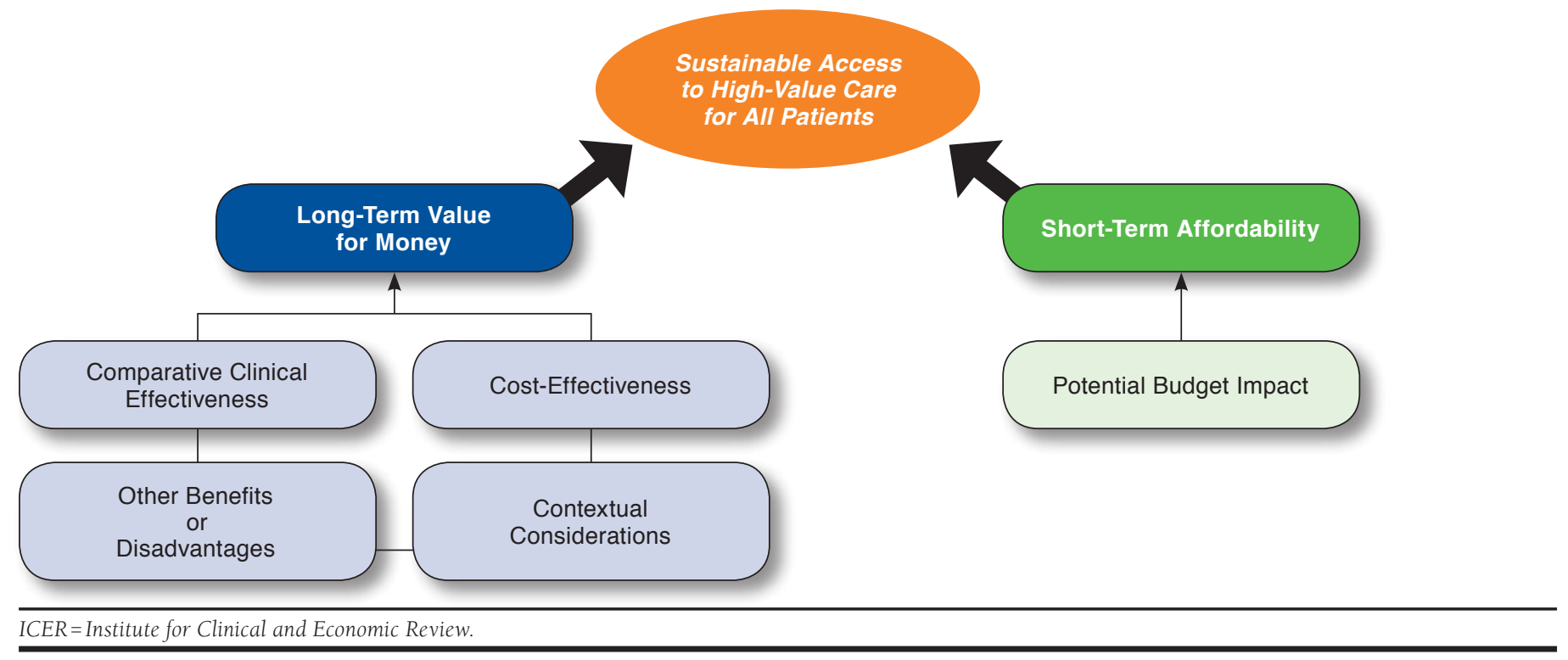

methodologists, and leaders in patient engagement and advocacy. Their deliberation includes input from clinical experts and patient representatives specific to the condition under review, as well as formal comment from manufacturers and the public. A policy roundtable concludes each meeting during which representatives from insurers and manufacturers join with clinical experts and patient representatives to discuss how best to apply the findings of the evidence to clinical practice, insurance coverage, and pricing negotiations.

The structure through which ICER evidence reports present information to the Midwest CEPAC is presented in Figure 1. This value assessment framework represents the conceptual framework through which considerations of different elements of value are integrated in judgments on long-term value for money and short-term affordability. ${ }^{28,29}$

The ICER report on inotersen and patisiran was the subject of a Midwest CEPAC meeting in September 2018. Following discussion, the CEPAC panel members voted that the evidence was adequate to demonstrate the superiority of both drugs versus standard of care: 9-2 for inotersen and 10-1 for patisiran. The panel also voted 10-1 that the evidence was not adequate to distinguish between the net health benefit provided by the 2 new treatments.

The CEPAC panel voted on "other potential benefits" and "contextual considerations" as part of a process intended to signal to policymakers whether there are important considerations when making judgments about long-term value for money not adequately captured in analyses of clinical and/or cost effectiveness. The results of these votes are shown in Tables 2 and 3 and serve to highlight several factors that the CEPAC panel felt were particularly important for judgments of value.

The culminating vote of the Midwest CEPAC, intended to reflect its integration of the relevant elements of the value assessment framework, was on the "long-term value for money" of inotersen and patisiran. The CEPAC panel members voted that for adults with hATTR, the long-term value for money (low, intermediate, or high) of these agents plus best supportive care compared with best supportive care alone is "low": 11-0-0 for inotersen and 11-0-0 for patisiran.

The policy roundtable discussion explored how best to translate the evidence and broader perspectives discussed into clinical practice and into pricing and insurance coverage policies. The full set of policy recommendations can be found in the Final Evidence Report ${ }^{5}$; however, key policy recommendations are as follows:

- Payers and other policymakers should consider potential broader benefits of inotersen and patisiran to patients, caregivers, and society, while simultaneously working to maintain affordability of health insurance for all patients now and in the future.

- Given the novel mechanisms of action, lack of long-term safety and efficacy data, and high cost, it is reasonable for insurers and other payers to develop prior authorization criteria to ensure prudent use of these treatments.

- Prior authorization criteria should be based on clinical evidence, with input from clinical experts and patient groups. Specific elements of coverage criteria for hATTR drugs are included in the final Evidence Report. ${ }^{5}$ 


\section{TABLE 2 Other Benefits or Disadvantages}

When compared to best supportive care alone, does the addition of inotersen or patisiran offer one or more of the following "other benefits"?

Potential Benefit

a. This intervention offers reduced complexity that will significantly improve patient outcomes

b. This intervention will reduce important health disparities across racial, ethnic, gender, socioeconomic, or regional categories

c. This intervention will significantly reduce caregiver or broader family burden

$\mathrm{d}$. This intervention offers a novel mechanism of action or approach that will allow successful treatment of many patients who have failed other treatments

e. This intervention will have a significant impact on improving patients' ability to return to work and/or their overall productivity

f. This intervention will have a significant positive impact outside the family, including on schools and/or communities

g. This intervention will have a significant impact on the entire "infrastructure" of care, including effects on screening for affected patients, on the sensitization of clinicians, and on the dissemination of understanding about the condition, that may revolutionize how patients are cared for in many ways that extend beyond the treatment itself

h. There are other important benefits or disadvantages that should have an important role in judgements of the value of this intervention

aEleven Midwest CEPAC panelists voted.

CEPAC = Comparative Effectiveness Public Advisory Council.

\section{TABLE 3 Contextual Considerations}

Are any of the following contextual considerations important in assessing inotersen's or patisiran's long-term value for money in patients?

Contextual Consideration

Panel Votes ${ }^{\mathrm{a}}$

a. This intervention is intended for the care of individuals with a condition of particularly high severity in terms of impact on length of life and/or quality of life

b. This intervention is intended for the care of individuals with a condition that represents a particularly high lifetime burden of illness

c. This intervention is the first to offer any improvement for patients with this condition

d. Compared to best supportive care, there is significant uncertainty about the long-term risk of serious side effects of this intervention

e. Compared to best supportive care, there is significant uncertainty about the magnitude or durability of the long-term benefits of this intervention

f. There are additional contextual considerations that should have an important role in judgements of the value of this intervention

aEleven MidWest CEPAC panelists voted.

CEPAC = Comparative Effectiveness Public Advisory Council

- Manufacturers should bring the price for innovative treatments for hATTR down to a level that aligns fairly with the added benefits for patients.

- Future research should address the durability of improvements in neurological function, longer-term safety, and cardiac outcomes provided by treatments for hATTR.

\section{Conclusions}

The RNA-based therapeutics patisiran and inotersen for hATTR represent an important advance in the treatment of a rare genetic disorder with high unmet need. However, despite consideration of potential broader benefits and of the contextual considerations associated with treatment of an ultra-rare disorder, the pricing of these drugs in the U.S. health care system was judged to represent low long-term value for money. Further efforts are needed to help align the price of these treatments with their demonstrated benefits in order to ensure sustainable access to high-value care for all patients.

\section{Authors}

KRISTIN MICKLE, MPH; WILLIAM B. DREITLEIN, PharmD, BCPS; and STEVEN D. PEARSON, MD, MSc, Institute for Clinical and Economic Review, Boston, Massachusetts. KAREN E. LASSER, MD, MPH, Boston University School of Medicine, Boston, Massachusetts; LAUREN E. CIPRIANO, PhD, Ivey Business School, Western University, London, Ontario, Canada; and JEFFREY S. $\mathrm{HOCH}, \mathrm{PhD}$, Center for Healthcare Policy and Research, University of California, Davis.

AUTHOR CORRESPONDENCE: William B. Dreitlein, PharmD BCPS, Institute for Clinical \& Economic Review, Two Liberty Square, 9th Fl., Boston, MA 02109. E-mail: bdreitlein@icer-review.org.

\section{DISCLOSURES}

Funding for this summary was contributed by the Laura and John Arnold Foundation, Blue Shield of California, and California Health Care Foundation to the Institute for Clinical and Economic Review (ICER), an independent organization that evaluates the evidence on the value of health care interventions. ICER's annual policy summit is supported by dues from Aetna, AHIP, 
Anthem, Blue Shield of California, CVS Caremark, Express Scripts, Harvard Pilgrim Health Care, Cambia Health Solutions, United Healthcare, Kaiser Permanente, Premera Blue Cross, AstraZeneca, Genentech, GlaxoSmithKline, Johnson \& Johnson, Merck, National Pharmaceutical Council, Prime Therapeutics, Sanofi, Spark Therapeutics, Health Care Service Corporation, Editas, Alnylam, Regeneron, Mallinkrodt, Biogen, HealthPartners, and Novartis. Mickle, Dreitlein, and Pearson are ICER employees. Lasser, Cipriano, and Hoch have nothing to disclose.

\section{ACKNOWLEDGMENTS}

The authors thank Rick Chapman and Dan Ollendorf for their contributions to ICER's hATTR report and Midwest CEPAC public meeting and David Whitrap and Alexandra Ellis for their review of this manuscript.

\section{REFERENCES}

1. Gertz MA. Hereditary ATTR amyloidosis: burden of illness and diagnostic challenges. Am J Manag Care. 2017;23(7 Suppl):S107-S112.

2. Hawkins PN, Ando Y, Dispenzeri A, Gonzalez-Duarte A, Adams D, Suhr OB. Evolving landscape in the management of transthyretin amyloidosis. Ann Med. 2015:47(8):625-38.

3. Adams D, Gonzalez-Duarte A, O'Riordan WD, et al. Patisiran, an RNAi therapeutic, for hereditary transthyretin amyloidosis. New Engl J Med. 2018;379(1):11-21.

4. Quarta CC, Buxbaum JN, Shah AM, et al. The amyloidogenic V122I transthyretin variant in elderly black Americans. New Engl J Med. 2015;372(1):21-29

5. Lasser K, Hoch JS, Mickle K, et al. Inotersen and patisiran for hereditary transthyretin amyloidosis: effectiveness and value. Final evidence report. Institute for Clinical and Economic Review. October 4, 2018. Available at: https://icer-review.org/wp-content/uploads/2018/02/ICER_Amyloidosis_ Final_Evidence_Report_100418.pdf. Accessed December 4, 2018.

6. Benson MD, Waddington-Cruz M, Berk JL, et al. Inotersen treatment for patients with hereditary transthyretin amyloidosis. New Engl J Med. 2018;379(1):22-31

7. Berk J. Safety and efficacy of inotersen in patients with hereditary transthyretin amyloidosis with polyneuropathy (NEURO-TTR). Paper presented at: American Academy of Neurology 2018 Annual Meeting; April 21-27, 2018; Los Angeles, CA.

8. Coehlo T, Vita G, Polydefkis M, et al. Inotersen improved Norfolk Quality of Life-Diabetic Neuropathy MEasures in patients with hereditary transthyretin amyloidosis treated in the phase 3 study NEURO-TTR. Paper presented at: 4th Congress of the European Academy of Neurology 2018; June 16-19, 2018; Lisbon, Portugal.

9. Benson MD, Dasgupta NR, Rissing SM, Smith J, Feigenbaum H. Safety and efficacy of a TTR specific antisense oligonucleotide in patients with transthyretin amyloid cardiomyopathy. Amyloid. 2017;24(4):219-25.

10. Tegdesi (inotersen) injection, for subcutaneous use. Carlsbad CIP Revised October 2018. Available at: https://www.accessdata.fda.gov/drugsatfda_docs/label/2018/211172lbl.pdf. Accessed December 4, 2018.

11. Adams D, Gonzalez-Duarte A, O'Riordan W, et al. Patisiran, an investigational RNAi therapeutic for patients with hereditary transthyretin-mediation (hATTR) amyloidosis: results from the phase 3 APOLLO study.

Paper presented at: American Academy of Neurology 2018 Annual Meeting; April 21-27, 2018. Los Angeles, CA.

12. Adams D, Gonzalez-Duarte A, O'Riordan W, et al. Evaluation of quality of life and disability in patients with hereditary transthyretin-mediated (hATTR) amyloidosis with polyneuropathy following treatment with patisiran, an investigational RNAi therapeutic: results from the phase 3 APOLLO study. Paper presented at: American Academy of Neurology 2018 Annual Meeting; April 21-27, 2018; Los Angeles, CA.
13. Adams D, Gonzalez-Duarte A, O'Riordan W, et al. Patisiran, an investigational RNAi therapeutic for the treatment of hereditary attr amyloidosis with polyneuropathy: results from the phase 3 APOLLO study. Paper presented at: European ATTR Amyloidosis Meeting for Patients and Doctors; November 2-3, 2017; Paris, France.

14. Obici L, Coehlo T, Gonzalez-Duarte A, et al. Impact of patisiran, an investigational RNAi therapeutic, on nutritional status in patients with hereditary transthyretin-mediated amyloidosis. Paper presented at: 4th Congress of the European Academy of Neurology 2018; June 16-19, 2018; Lisbon, Portugal.

15. Slama M, Solomon S, Adams D, et al. Analysis of NT-proBNP baseline levels in APOLLO as a predictor of survival in hereditary transthyretinmediated (hATTR) amyloidosis. Paper presented at: European Society of Cardiology Heart Failure 2018 Congress; May 26-29, 2018; Vienna, Austria.

16. Kristen A, Solomon S, Adams D, et al. APOLLO a phase 3 study of patisiran for the treatment of hereditary transthyretin-mediated amyloidosis; 18-month safety and efficacy in subgroup of patients with cardiac involvement. Paper presented at: 16th International Symposium on Amyloidosis; March 26-29, 2018; Kumamoto, Japan.

17. Gonzalez-Duarte A, Adams D, O'Riordan W, et al. Changes in neuropathy stage in patients with hereditary transthyretin-mediated amyloidosis following treatment with patisiran, an investigational RNAi therapeutic: an analysis from the phase 3 APOLLO study. 16th International Symposium on Amyloidosis; March 26-29, 2018; Kumamoto, Japan.

18. Merlini G, Solomon S, Adams D, et al. Impact of patisiran on Norfolk Quality of Life Questionnaire Diabetic Neuropathy in patients with hereditary transthyretin-mediated amyloidosis: results from the cardiac subpopulation in the phase 3 APOLLO study. Paper presented at: European Society of Cardiology Heart Failure 2018 Congress; May 26-29, 2018; Vienna, Austria.

19. Suhr OB, Coelho T, Buades J, et al. Efficacy and safety of patisiran for familial amyloidotic polyneuropathy: a phase II multi-dose study. Orphanet J Rare Dis. 2015;10:109.

20. Alnylam Pharmaceuticals. Patisiran, an investigational RNAi therapeutic for the treatment of hereditary transthyretin-mediated (hATTR) amyloidosis [press release]. August 3, 2017. Available at: https://www.alnylam.com/ wp-content/uploads/2017/08/2017-0803-TTR-RNAi-Roundtable-FINAL.pdf. Accessed December 18, 2018.

21. Adams D, Coelho T, Conceicao I, et al. Phase 2 open-label extension (OLE) study of patisiran with or without a TTR stabilizer for the treatment of hereditary ATTR (hATTR) amyloidosis with polyneuropathy [abstract]. Eur J Neurol. 2017;24(Suppl 1):31-32. Available at: https://onlinelibrary.wiley, com/doi/epdf/10.1111/ene.13366. Accessed December 4, 2018.

22. Adams D, Coelho T, Conceicao I, et al. Phase 2 open-label extension (OLE) study of patisiran, an investigational RNA interference (RNAi) therapeutic for the treatment of hereditary ATTR amyloidosis with polyneuropathy. [abstract] Value Health. 2017;20(5):A211-A212.

23. Suhr O, Gonzalez-Duarte A, O'Riordan W, et al. Long-term use of patisiran, an investigational RNAi therapeutic, in patients with hereditary transthyretin-mediated (hATTR) amyloidosis: baseline demographics and interim data from global open label extension. Paper presented at: 16th International Symposium on Amyloidosis; March 26-29, 2018; Kumamoto, Japan.

24. Gillmore JD, Suhr O, Hazenberg B, et al. Home infusion administration of patisiran, an investigational RNAi therapeutic in patients with hereditary transthyretin-mediated amyloidosis: an analysis of safety and adherence. 16th International Symposium on Amyloidosis; March 26-29, 2018; Kumamoto, Japan.

25. Solomon S, Adams D, Kristen A, et al. Effects of patisiran, an RNA interference therapeutic, on cardiac parameters in patients with hereditary transthyretin-mediated amyloidosis: an analysis of the APOLLO study. Circulation. September 14, 2018 [Epub ahead of print]. Available at: https:// www.ahajournals.org/doi/10.1161/CIRCULATIONAHA.118.035831. Accessed December 4, 2018. 
26. Institute for Clinical and Economic Review. Modifications to the ICER value assessment framework for treatments for ultra-rare diseases. November 2017. Available at: https://icer-review.org/wp-content/ uploads/2017/11/ICER-Adaptations-of-Value-Framework-for-Rare-Diseases. pdf. Accessed December 4, 2018.

27. Bloomberg. FDA approves $\$ 450,000$-a-year treatment for rare disease. Fortune. August 11, 2018. Available at: http://fortune.com/2018/08/11/fdaalnylam-oppattro-approved/2018. Accessed December 4, 2018.
28. Pearson SD. The ICER value framework: integrating cost effectiveness and affordability in the assessment of health care value. Value Health. 2018;21(3):258-65.

29. Institute for Clinical and Economic Review. ICER value assessment framework. Available at: https://icer-review.org/methodology/icers-methods/ icer-value-assessment-framework/. Accessed December 4, 2018.

\title{
TTR Gene Silencers for Hereditary ATTR Amyloidosis: More than ICER Recognized
}

\author{
John L. Berk, MD
}

\section{COMMENTARY}

13, 2018, the Institute for Clinical and Economic Review (ICER), a nonprofit research organization created to provide impartial reviews and economic guidance to parties interested in new treatments approved by the U.S. Food and Drug Administration (FDA), presented its findings on 2 transthyretin (TTR) gene-silencing drugs to the Midwest Comparative Effectiveness Public Advisory Council (Midwest CEPAC), a committee of medical evidence experts. The products of billion-dollar investments by Alnylam Pharmaceuticals (Cambridge, MA) and Ionis Pharmaceuticals/Akcea Therapeutics (Carlsbad, CA) received grades of $\mathrm{B}+$ and $\mathrm{C}+$, respectively, on the scale of quality-adjusted life-years. For patients with hereditary transthyretin amyloidosis (hATTR) and their advocacy organizations committed to getting drugs to affected people, the lukewarm grading was surprising and disappointing.

Two months earlier, the New England Journal of Medicine featured 2 lead articles and an editorial, ${ }^{1-3}$ trumpeting diseasealtering randomized controlled trial (RCT) results for these TTR gene-silencing drugs-patisiran, an RNA interference agent, and inotersen, an antisense oligonucleotide drug-in the treatment of hATTR. hATTR is an autosomal dominant lethal neurodegenerative disease resulting from the misaggregation and organ deposition of mutated transthyretin. Advancing 10 times as quickly as diabetic polyneuropathy, patients with untreated hATTR amyloid polyneuropathy die 5-15 years after diagnosis from progressive loss of limb function, bowel control, and blood pressure regulation that renders them bed bound and completely dependent on others. It is a disease that devastates the afflicted and traumatizes family caregivers.

\section{J Manag Care Spec Pharm. 2019;25(1):15-16}

Copyright $\odot 2019$, Academy of Managed Care Pharmacy. All rights reserved.
The positive results of the patisiran and inotersen studies were preceded 5 years earlier by 2 RCTs that demonstrated the ability of TTR protein stabilizers to inhibit TTR misfolding and slow neurologic decline in patients with hATTR amyloidosis. ${ }^{4,5}$ Those drugs, diflunisal (generic nonsteroidal anti-inflammatory drug) and tafamidis (FoldRx/Pfizer), changed the lives of many patients. Despite an overall beneficial effect, however, over $75 \%$ of patients treated with TTR protein stabilizers experience continued neurologic decline. In contrast, the TTR gene silencers more reliably halt disease and, for the first time, offer potential improvement in polyneuropathy and, possibly, heart function in $37 \%-56 \%$ of those receiving treatment. ${ }^{1,2}$ Moreover, an ad hoc analysis report showed a decrease in all-cause mortality and cardiac hospitalization rates in patients randomized to the TTR gene silencer patisiran versus placebo patients. ${ }^{6}$

Why then the disparate findings and the lukewarm ICER/ Midwest CEPAC grading of TTR gene silencers?

To the external observer, the ICER time line from data review to drug grading appeared overly ambitious. Six months before the September 2018 Midwest CEPAC presentations, ICER posted scoping documents for the 2 topics on the meeting agenda: (a) use of antiandrogen therapies in nonmetastatic prostate cancer and (b) effectiveness of TTR gene silencers in hATTR amyloid neuropathy. However, full access to RCT data critical to ICER's proposed analyses and presentations did not occur until the data embargo imposed by the New England Journal of Medicine was lifted with the publication of study results for the antiandrogen products on April 12, 2018, and the publication of trial data for the TTR gene silencers on July 5 , 2018, nearly 3 months later. ${ }^{7,8}$ Despite the relative inaccessibility of the TTR gene silencing trial data, evidence reports for antiandrogen and TTR gene-silencer presentations were posted 